\title{
Reconstruction of the Regulatory Network for Bacillus subtilis and Reconciliation with Gene Expression Data
}

\author{
José P. Faria ${ }^{1,2,3 *}$, Ross Overbeek ${ }^{4}$, Ronald C. Taylor ${ }^{5}$, Neal Conrad ${ }^{2}$, Veronika Vonstein ${ }^{4}$, \\ Anne Goelzer ${ }^{6}$, Vincent Fromion ${ }^{6}$, Miguel Rocha ${ }^{3}$, Isabel Rocha ${ }^{3}$ and \\ Christopher S. Henry ${ }^{1,7 *}$ \\ ${ }^{1}$ Computation Institute, University of Chicago, Chicago, IL, USA, ${ }^{2}$ Computing, Environment and Life Sciences, Argonne \\ National Laboratory, Argonne, IL, USA, ${ }^{3}$ Centre of Biological Engineering, University of Minho, Braga, Portugal, ${ }^{4}$ Fellowship \\ for Interpretation of Genomes, Burr Ridge, IL, USA, ${ }^{5}$ Computational Biology and Bioinformatics Group, Pacific Northwest \\ National Laboratory, United States Department of Energy, Richland, WA, USA, ${ }^{6}$ UR1404 Applied Mathematics and \\ Computer Science from Genomes to the Environment, INRA, Paris-Saclay University, Jouy-en-Josas, France, ${ }^{7}$ Mathematics \\ and Computer Science Division, Argonne National Laboratory, Argonne, IL, USA
}

\section{OPEN ACCESS}

Edited by: Jonathan Michael Plett, Western Sydney University, Australia

Reviewed by: Paul Richard Himes, University of Louisville, USA Stephanie A. Smith, Washington State University, USA

*Correspondence: Christopher S. Henry chenry@mcs.anl.gov José P. Faria jplfaria@uchiago.edu

Specialty section: This article was submitted to Systems Microbiology, a section of the journal Frontiers in Microbiology

Received: 01 December 2015 Accepted: 19 February 2016 Published: 18 March 2016

Citation:

Faria JP, Overbeek R, Taylor RC, Conrad N, Vonstein V, Goelzer A,

Fromion V, Rocha M, Rocha I and Henry CS (2016) Reconstruction of the Regulatory Network for Bacillus subtilis and Reconciliation with Gene Expression Data.

Front. Microbiol. 7:275 doi: 10.3389/fmicb.2016.00275
We introduce a manually constructed and curated regulatory network model that describes the current state of knowledge of transcriptional regulation of Bacillus subtilis. The model corresponds to an updated and enlarged version of the regulatory model of central metabolism originally proposed in 2008. We extended the original network to the whole genome by integration of information from DBTBS, a compendium of regulatory data that includes promoters, transcription factors (TFs), binding sites, motifs, and regulated operons. Additionally, we consolidated our network with all the information on regulation included in the SporeWeb and Subtiwiki community-curated resources on B. subtilis. Finally, we reconciled our network with data from RegPrecise, which recently released their own less comprehensive reconstruction of the regulatory network for B. subtilis. Our model describes 275 regulators and their target genes, representing 30 different mechanisms of regulation such as TFs, RNA switches, Riboswitches, and small regulatory RNAs. Overall, regulatory information is included in the model for $\sim 2500$ of the $\sim 4200$ genes in B. subtilis 168. In an effort to further expand our knowledge of $B$. subtilis regulation, we reconciled our model with expression data. For this process, we reconstructed the Atomic Regulons (ARs) for B. subtilis, which are the sets of genes that share the same "ON" and "OFF" gene expression profiles across multiple samples of experimental data. We show how ARs for B. subtilis are able to capture many sets of genes corresponding to regulated operons in our manually curated network. Additionally, we demonstrate how ARs can be used to help expand or validate the knowledge of the regulatory networks by looking at highly correlated genes in the ARs for which regulatory information is lacking. During this process, we were also able to infer novel stimuli for hypothetical genes by exploring the genome expression metadata relating to experimental conditions, gaining insights into novel biology.

Keywords: Atomic Regulon, regulatory network, stimuli, regulation, Bacillus subtilis 


\section{INTRODUCTION}

Proper elucidation and characterization of gene regulatory networks has become one of the major challenges of the postgenomic era (Medini et al., 2008). Genome-wide studies of the transcriptome of the minimal organism Mycoplasma pneumoniae (Guell et al., 2009) and the model organism Escherichia coli (Cho et al., 2009) both reveal a complex regulatory architecture. This complexity can be attributed to the diversity of regulatory mechanisms in bacteria, such as transcription factors (TFs), RNA switches (Mironov et al., 2002), antisense RNA (Wagner and Simons, 1994), small RNAs (Chen and Rajewsky, 2007), or riboswitches (Nudler and Mironov, 2004). Our focus organism, Bacillus subtilis, is most commonly found in soil, and is subject to a wide variety of external environmental conditions (Illing, 2002). This reinforces the importance of understanding the regulatory mechanisms that allow the $B$. subtilis bacterium to survive and adapt to such conditions.

As a model organism, literature for B. subtilis regulation is extensive and several resources/databases are available. A regulatory network model for the central carbon metabolism was made available by Goelzer et al. (2008). Multiple inferred networks based on expression data have also been proposed in the literature (de Hoon et al., 2003; Steggles et al., 2007; Fadda et al., 2009). RegPrecise (Novichkov et al., 2010a), a resource for transcription factor binding site (TFBS) based network inference also provides a network for B. subtilis (Leyn et al., 2013). Subtiwiki (Florez et al., 2009; Michna et al., 2014) is a community collaborative resource for $B$. subtilis that includes a vast compendium of regulatory information. DBTBS (Sierro et al., 2008) is another B. subtilis comprehensive resource of regulatory data with promoters, TFs, TFBS, motifs and regulated operons. Our novel genome-scale reconstruction of the B. subtilis regulatory network integrates the previous work from the Goelzer et al. (2008) literature and the other notable resources for regulation described above (Sierro et al., 2008; Florez et al., 2009; Novichkov et al., 2010a; Mader et al., 2012).

We reconciled our new model against a large set of highquality gene expression data (Buescher et al., 2012; Nicolas et al., 2012). For the process of reconciliation with expression data, we introduce the concept of Atomic Regulons (ARs). ARs are sets of co-regulated genes that share the same "ON" and "OFF" expression profile (meaning the genes in these sets are "ON" and "OFF" in the same conditions) (submitted). Our construction of ARs began by predicting draft regulons using a combination of crude operon predictions and SEED subsystem technology (Ermolaeva et al., 2001; Overbeek et al., 2005; Price et al., 2005). We then decompose and expand these draft regulons based on consistency with expression data. This process results in the set of co-regulated gene clusters that we call ARs. We show how ARs can be used to help expand/validate the knowledge of the B. subtilis regulatory network.

\section{MATERIALS AND METHODS}

In this work, we explore the reconstruction of regulatory networks using two different approaches. In a first approach, we combine the information available in databases with notable regulatory transcriptional data for $B$. subtilis into a comprehensive manually curated regulatory network. In the second approach, we developed a methodology, dubbed "AR inference", to infer regulatory interactions from a combination of gene expression data, predicted operons, and SEED subsystems-based annotations. For this purpose, we chose a dataset of expression data comprised of 269 samples across 104 different experimental conditions (Buescher et al., 2012; Nicolas et al., 2012). We selected this dataset for its remarkable quality, consistency, and coverage. All work to produce this dataset was conducted according to a pre-agreed Standard Operating Procedure, and only $4.4 \%$ of the known CDS in the selected strain were not expressed in any tested conditions. The whole data set is deposited in the NCBI Gene Expression Omnibus (GEO) under the accession number GSE27219. All of the different experimental conditions were performed using the BaSysBio ${ }^{1}$ reference strain BSB1. This strain is a tryptophan-prototrophic (trp +$)$ derivative of the B. subtilis 168 trpC2 strain (Anagnostopoulos and Spizizen, 1961). Finally, to take advantage of both approaches and expand our knowledge of the B. subtilis regulatory network, we propose a process to reconcile their output.

We define an AR as a set of genes with identical binary (ON/OFF) expression profiles. That is, all the genes included in the same AR will always have the same state: either "all ON" or "all OFF". This notion has meaning only in a simplified model of the cell in which genes are either ON or OFF in any condition. Thus, we must have the ability to accurately assign genes to these binary states based on their normalized expression values from a variety of experimental samples.

Atomic Regulons differ subtly from existing abstractions for describing the co-regulation of genes: regulons (set of genes that respond to the same regulator), and stimulons (set of genes that respond to the same stimuli) (Figure 1). Figure 1A shows a set of six genes (G1-G6) being regulated by three regulators (R1-R3) and effected by two stimuli (S1 and S2). Figure 1B overlays the theoretical ARs with the previous figure.

\section{Atomic Regulon Curation}

Genes contained within the same AR share a common expression profile; so we assume they respond to the same stimuli. Following that principle, three assertions were made for the reconciliation of our manually curated network with expression data:

(1) Each regulon is a subset of at least one stimulon;

(2) Genes often take part in multiple stimulons, and they will vary in whether they are induced (expression increases) or suppressed (expression decreases) in the stimulon;

(3) A set of genes that all take part in identical sets of stimulons with identical induction/suppression profiles comprises an AR.

\footnotetext{
${ }^{1}$ http://www.basysbio.eu/
} 


\section{A Stimulons and Regulons}

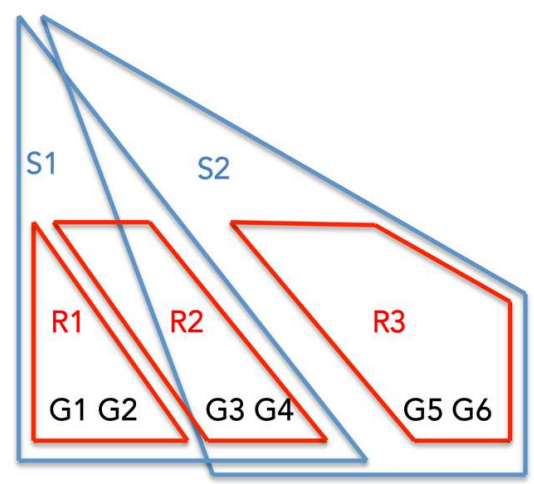

B Atomic Regulons in context with Stimulons and Regulons

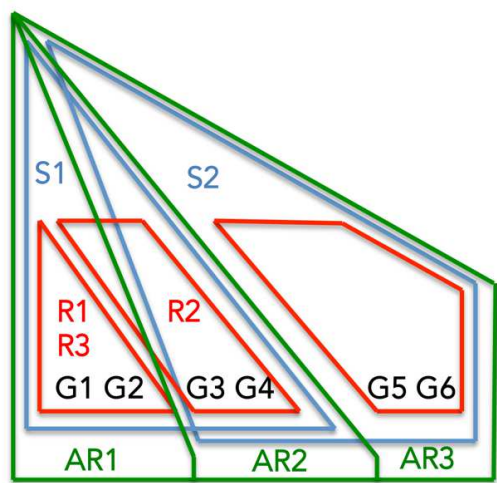

FIGURE 1 | The interplay between Stimulons, Regulons, and Atomic Regulons (ARs). (A) The representation features six genes (G1-G6), three regulators (R1-R3), and two stimuli (S1-S2). The red lines define the regulons and the blue lines define the stimulons. (B) Features in addition to (A): the Atomic Regulons (AR1-AR3) in the representation as sets of genes that have identical binary expression profiles.

Figure 1B demonstrates these criteria: AR1 includes genes only affected by S1, AR2 includes genes affected by S1 and S2, and AR3 includes genes only affected by S2.

A set of comparative genomics tools was used in the manual curation of the ARs. In this curation, we attempted to ensure the conservation of chromosomal gene synteny among colocalized members of an AR over phylogenetic distance. To conduct our curation, we used the "Compare regions tool" within the SEED environment (Overbeek et al., 2005). We also verified the functional annotation of $A R$ members using comparative genomics tools like the "Compare regions tool" or the "Alignment and Tree tool" of the SEED environment. We also conducted BLASTp searches against NCBI's conserved domain database (CDD) (Marchler-Bauer et al., 2015).

\section{The Web Resource}

We developed a set of web tools to visualize all ARs we constructed for B. subtilis, along with the associated expression profiles and experimental metadata. The web tools are available at http://tinyurl.com/AtomicRegulons. The initial page provides the user with a list of the ARs, some of which have general descriptions.

The website can be used for other analyses of the expression data. That is, AR data presented on the web site for many different species can be used for studies outside the scope the work presented in this manuscript.

\section{RESULTS AND DISCUSSION}

\section{Draft Regulatory Network of Bacillus subtilis from Manual Curation}

We introduce a manually constructed and curated model that describes the current state of knowledge of the transcriptional network of $B$. subtilis. The model corresponds to an updated and enlarged version of the regulation network in the central metabolism originally proposed in Goelzer et al. (2008). We have firstly extended that original network to the whole genome by including the information from the DBTBS database (Sierro et al., 2008). The DBTBS compendium of regulatory data includes promoters, TFs, TFBS, motifs, and regulated operons. The addition of the DBTBS led to a significant increase in the size of the regulatory network (Table 1). Additionally, we consolidated our network with all the information on regulation included in SporeWeb (Eijlander et al., 2014) and in Subtiwiki (Florez et al., 2009; Mader et al., 2012) as of March 2013. Subtiwiki is the reference community-curated resource for $B$. subtilis that was integrated into the 2012 release of DBTBS. This consolidation with Subtiwiki resulted in some revision of regulatory data included in the original network by Goelzer et al. (2008). Also, it significantly enlarged the network with respect to other microbial processes. All the above data reflect experimentally validated regulatory interactions. Additionally, RegPrecise (Novichkov et al., 2010a), a database that provides tools (Novichkov et al., 2010b) for prediction and curation of regulons, recently released their reconstruction of the regulatory

TABLE 1 | Comparison between notable resources for Bacillus subtilis regulatory network modeling.

\begin{tabular}{|c|c|c|c|c|c|}
\hline Resource & TFs & Sigma factors & RNA regulators & Effectors & Regulated genes \\
\hline Goelzer et al., 2008 & 65 & 9 & 21 & 95 & 434 \\
\hline Leyn et al., 2013 & 129 & - & 33 & 130 & 1065 \\
\hline This work & 175 & 19 & 60 & 169 & 2570 \\
\hline
\end{tabular}


network for B. subtilis (Leyn et al., 2013). Reconciliation with the RegPrecise inferred network resulted in the addition of a total of 39 regulators and their target gene sets to our experimentally validated network.

We compared the reconstruction with previously described reconstructions in the literature (Table 1). This comparison shows a substantial increase in network coverage from the original Goelzer et al. (2008) This increase is due in large part to an expansion of the scope of our model from the central carbon metabolism to genome-scale, as well as our effort to include most of the regulation mechanisms for $B$. subtilis that have been described in the literature to date. Our model includes $175 \mathrm{TF}$ regulators, representing a wide variety of regulatory mechanisms: TFs conditioned by metabolites, accessory proteins, phosphorylated proteins, and stress factors. Sigma factor regulation was also included as it plays a role in governing many major cell functions such as sporulation (sigE, sigF, sigG, and sigK), regulation of flagella, motility, and chemotaxis (sigD), cell wall surface properties, and stress (sigX, sigW, and sigV). Elements relating to anti-sense RNA, riboswitches, RNA switches, RNA antiterminators, and small regulatory RNAs compose the 60 RNA regulators described in our network.

The increase in the number of regulators in our model led to a corresponding increase in effectors. We divide our effectors into two categories: biochemical (involving metabolites), and environmental effectors (e.g., DNA damage and heat shock). The 275 regulators in our model are linked to a set of regulons comprised of $\sim 2500$ genes. However, notably, the detailed regulatory mechanisms associated with some of the regulons in our model, particularly in cases of sigma factor and RNA regulation, remain unclear, or unknown. All details related to the regulatory model, including data sources are provided in Supplementary Tables S1 and S2. An online version is also available at http://modelseed.org/projects/regulons.

We first classified the 275 regulators in our model with respect to their associated regulatory mechanism (Figure 2A). Some regulatory mechanisms were not completely known. We thus introduced additional categories such as TFs conditioned by an unknown regulatory mechanism ("TF+unk") to indicate the partial level of knowledge on these mechanisms (see Supplementary Table $\mathbf{S 1}$ for the full list and description of categories). $40 \%$ of the regulators have a mechanism that directly responds to a metabolic signal (categories TFs conditioned by a metabolite (“TF+M"), TFs + phosphorylated protein + metabolite (“TF $+\mathrm{PP}+\mathrm{M}$ ”), TFs + accessory protein associated to a metabolite ("TF $+\mathrm{P}+\mathrm{M}$ ”), Protein - transcriptional antiterminator conditioned by a PTS phosphorylation ("P-AT + PTS"), Protein - transcriptional antiterminator conditioned by a metabolite ("P-AT $+\mathrm{M}$ "), riboswitch). In particular, the main regulatory mechanism ("TF $+\mathrm{M}$ ", associated with $25 \%$ of the regulators) involves a TFs having one (or several) metabolite(s) as direct effectors. These results emphasized the work of Goelzer et al. (2008) that originally pointed out the weight of metabolism in the genetic regulation of metabolic pathways. Metabolites are strongly involved in the activation of regulators at genome-scale.
We then examined the number of genes responding to the regulators associated with each distinct regulatory mechanism (Figure 2B). In agreement with the experimental results of (Nicolas et al., 2012), we found the largest number of genes were associated with mechanisms involving a sigma factor (Sigma factor regulatory mechanism is not picture in Figure $\mathbf{2 B}$ due to the disproportionally large number of genes associated with this mechanism, all details are available in Supplementary Tables S1 and S2). All together, these mechanisms were associated with $40 \%$ of the genes included in our model. Moreover, $26 \%$ of the genes in our model were associated with regulatory mechanisms that respond to a metabolic signal (categories " $\mathrm{TF}+\mathrm{M}$ ", “ $\mathrm{TF}+\mathrm{PP}+\mathrm{M}$ ”, "TF+P + M", "P-AT+PTS", "P-AT+M", "riboswitch"); this set of genes includes $45 \%$ of the genes involved in the metabolic pathways of B. subtilis (Henry et al., 2009) (Figure 2B highlighted in red), in agreement with results from previous work (Goelzer et al., 2008). This confirms the key role of metabolites in the regulation of metabolic pathways.

In addition to sigma factors, the other main regulatory mechanisms, representing $\sim 29 \%$ of the reported regulatory interactions, were TFs alone ("TF") and the TFs having one (or several) metabolites as direct effectors (“TF+M") (Figure 2). Most of the regulators of the category " $\mathrm{TF}+\mathrm{M}$ " correspond to local regulators, following the definition of Goelzer et al. (2008): "the local regulator of a metabolic pathway guarantees the activation or the inhibition of genes as a function of an intermediate metabolite of the metabolic pathway". Interestingly, the complex regulatory mechanisms involving several entities, like a TFs and a phosphorylated protein with or without a metabolite ("TF+PP" and "TF+PP $+\mathrm{M}$ ") had only a single pleiotropic regulator with multiple target genes. These regulators are SpoOA and CcpA and are involved, respectively, in the cell fate decision (Lopez and Kolter, 2010) and in the management of carbon sources (Fujita, 2009). Increasing the complexity of the mechanism of regulation may reflect the necessity for the cell to integrate different signals or to have a large range of modulation in the gene expression.

\section{Atomic Regulons for Bacillus subtilis}

With the initial reconstruction of our new regulatory model of B. subtilis complete, we next applied gene expression data to reconcile and validate our model. This process began with a survey of the data present in current expression databases. As of May 2015, there were $~ 1750$ datasets in GEO (Edgar et al., 2002) related to $B$. subtilis strains. However, the utilization of all of these data in our model validation posed a challenge, as protocols vary from lab to lab, and various expression platforms can produce results that are difficult to merge. Thus, we selected a single dataset, comprised of 269 samples collected from 104 different growth conditions (Buescher et al., 2012; Nicolas et al., 2012). We used this data with our new algorithm to infer a set of ARs for the B. subtilis 168 genome (Figure 3 and Supplementary Table S3).

A total of 688 ARs were computed comprising 3168 genes ( $\sim 72 \%$ of the genome) (Figure 3A). We categorized these ARs according to their expression profile (Figure 3B): only 4\% (137) of the genes were always OFF in all conditions, while 17\% (523) 

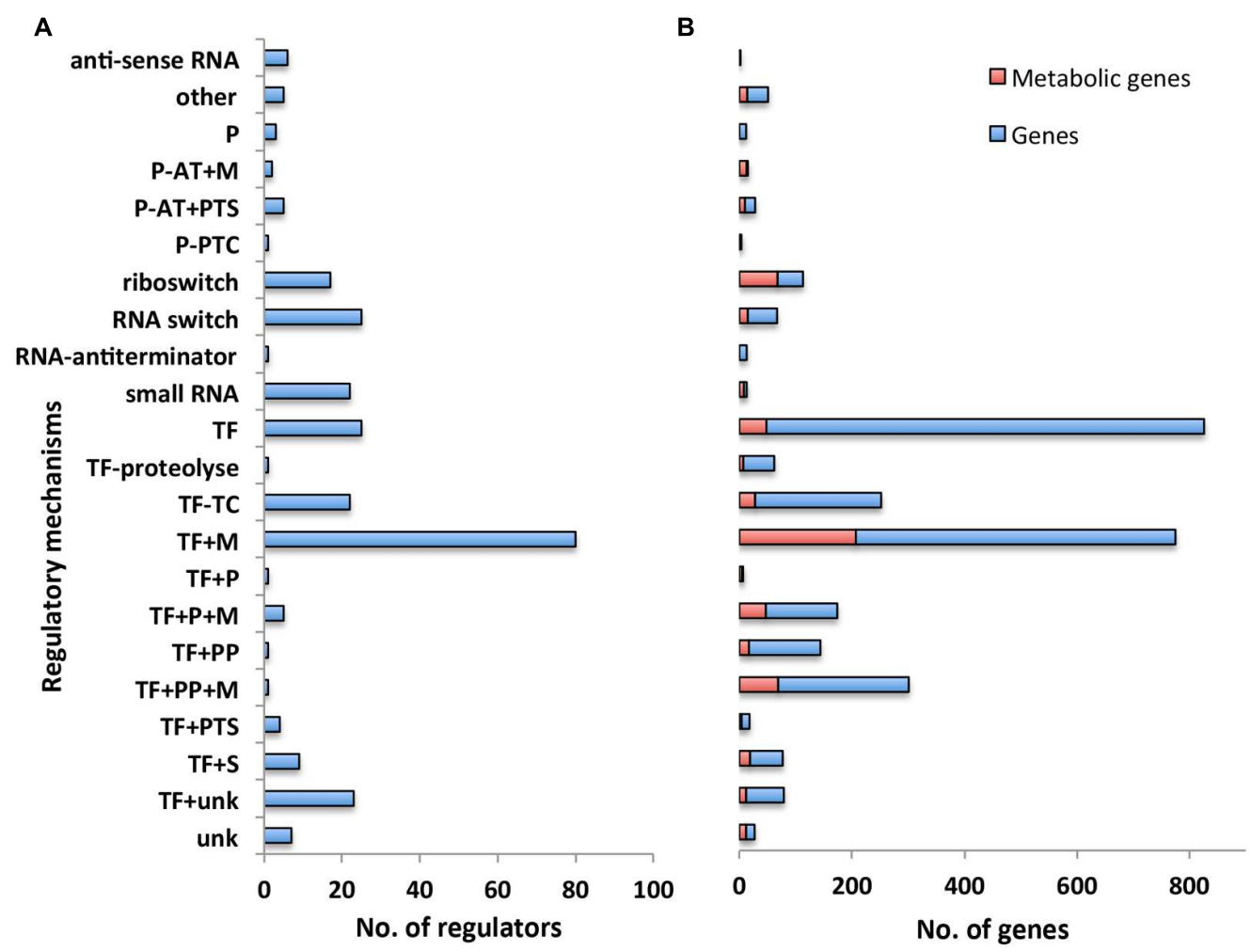

FIGURE 2 | Overview of regulatory network categorized by regulatory mechanism. (A) Number of regulators with respect to their regulatory mechanisms. Each bar corresponds to the number of regulators having the same type of regulatory mechanism. (B) Number of genes with respect to the regulatory mechanisms controlling their expression. Each bar refers to the number of genes that are controlled by a regulator having the same type of regulatory mechanism. Number of genes involved in the metabolism is highlighted in red. Abbreviations: P (Accessory protein involved in regulation); P-AT+M (Protein - transcriptional antiterminator conditioned by a metabolite); P-AT+PTS (Protein - transcriptional antiterminator conditioned by a PTS phosphorylation); P-PTC (Protein post-transcriptional control); TF (Transcription factor); TF-TC (Two-component response regulator); TF+M (Transcription factor conditioned by a metabolite); TF+P (Transcription factor + accessory protein); TF+P+M (Transcription factor + accessory protein associated to a metabolite); TF+PP (Transcription Factor + phosphorylated protein); TF+PP+M (Transcription factor + phosphorylated protein + metabolite); TF+PTS (Transcription factor + PTS phosphorylation); TF+S [Transcription factor + stress (DNA alteration/TF alteration)]; TF+unk (Transcription factor conditioned by an unknown mechanism/protein/metabolite).

of the genes were $\mathrm{ON}$ in all conditions. This result was consistent with the claim by the authors of the study that $95 \%$ of the genes in B. subtilis had been expressed in at least one condition. We explored the functions associated with the genes that we found to be always ON or always OFF (Figure 3C).

Forty percent of the always-ON genes (211) are categorized as information processing, which encompasses: RNA synthesis and degradation (transcription); protein folding, modification and degradation and translation; and, DNA replication. 25\% (129) of the always-ON genes were metabolic, including central carbon, nucleotide, and lipid metabolism. Finally, 12\% (63) of the always-ON genes were associated with cellular process, including cell wall biosynthesis, cell division, transporters and homeostasis.

The small set of genes (137) found to be OFF in all conditions is comprised of genes across a diverse set of functions. To verify that no gene found to be OFF in all conditions was an essential gene, we compared the set with a list of $B$. subtilis essential genes (Kobayashi et al., 2003; Commichau et al., 2013). No essential B. subtilis genes were found to be OFF in all conditions.

\section{Reconciling Expression Data with the Draft Regulatory Network for Bacillus subtilis}

Our definition of AR states that genes contained within the same AR must respond to the same set of stimuli (Figure 1). We can use this principle to identify and reconcile inconsistencies that exist between the stimuli mapped to the genes in our B. subtilis model and the set of genes comprising each AR. Considering sucrose as an example (Figure 4) we can explore the set of ARs computed for the genes comprising the Sucrose stimulon. We have eight genes in the Sucrose stimulon that respond to four distinct combinations of stimuli: (i) $y w d A$, sacA, and $s a c P$ all respond to both fructose-biphosphate and glucose-6-phosphate (Debarbouille et al., 1990); (ii) sacX and sacY respond to an uncharacterized stimulus (Tortosa and Le Coq, 1995); (iii) sacB and levB respond to two uncharacterized stimuli (Tortosa and Le Coq, 1995); and (iv) yveA shares the same uncharacterized stimuli as the previous genes, plus another uncharacterized stimulus (Pereira et al., 2001). 
A Atomic Regulon Inference from Expression Data

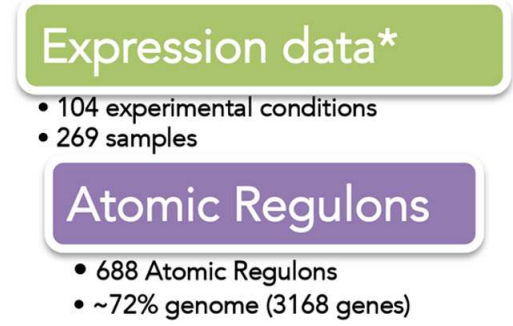

B Categorization of genes in Atomic Regulons

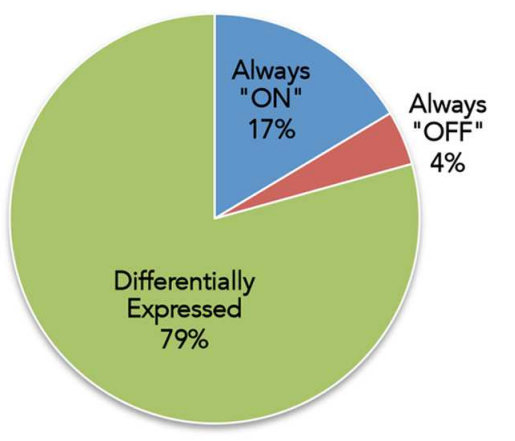

C Gene function for genes always "ON" and "OFF"

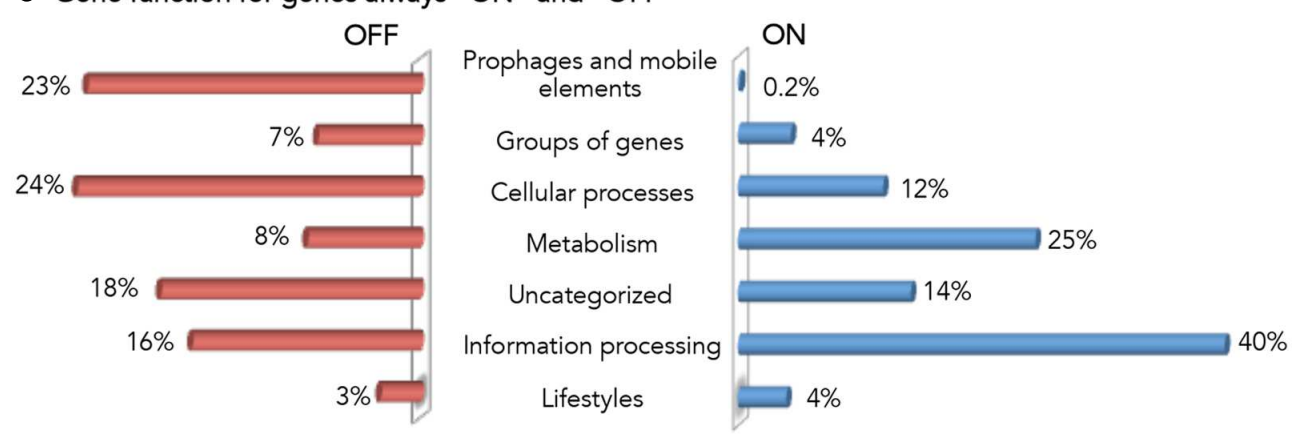

FIGURE 3 | Overview of Bacillus subtilis Atomic Regulons (ARs). (A) Expression data used for AR inference in B. subtilis (Buescher et al., 2012; Nicolas et al., 2012). (B) Categorization of genes in ARs. Genes have been categorized based on the expression profile as always "ON", always "OFF" and differentially expressed. (C) Gene function for genes always "ON" and "OFF". B. subtilis 168 genes are classified in SubtiWiki (Florez et al., 2009; Mader et al., 2012) into 6 categories cellular function; here we show the fraction of genes in the "always ON" regulon (right) and the "always OFF" regulon (left) that occur in each category.

Our AR inference algorithm divided the sucrose stimulon into three ARs (Table 2 and Figure 4): (i) AR \#376 containing $y w d A$, $s a c A$, and sacP, (ii) AR \#254 containing sacX and sacY; and (iii) AR \#625 containing sacB and levB. yveA was not placed into an $A R$ since the methodology requires two or more genes for placement in AR. The capacity of our AR inference methodology to divide these genes into separate ARs that conform perfectly with their distinctive stimuli demonstrates the robustness of the approach. Two of these ARs, \#254 and \#625, respond to uncharacterized effectors in our model (Figure 4A), and these ARs are active in no more than five and three experiments in our expression dataset respectively (Figure 4B). For AR 625 we checked the experiments in which the genes were ON (Table 3). Genes in AR 625 were found to be "ON" in experiments that tested gene expression at regular intervals after sporulation was induced. A detailed description of the study can be found by looking at the associated study number (in this case "study0003"2). Sporulation was induced with the use of CH medium (Sterlini and Mandelstam, 1969), and cells were harvested at hourly intervals, with the genes in our ARs being $\mathrm{ON}$ in the late intervals of the study. This fact tells us that our uncharacterized effector can be related with sporulation and that a compound in the $\mathrm{CH}$ medium can be a candidate for the uncharacterized effector.

\footnotetext{
${ }^{2}$ http://tinyurl.com/ARStudies
}

To assess the consistency of all ARs when compared with the stimuli in the regulatory network, we organized all computed ARs into four categories: consistent; consistent with missing stimuli; inconsistent; and empty (counts in Table 4). The consistent category comprises ARs with members that have the same stimuli/effectors in the regulatory network. The consistent with missing stimuli category comprises cases in which a member of the AR has the same stimuli/effectors, while other members have no described stimuli in the regulatory network. The inconsistent category displays ARs with members that have different stimuli associated in the regulatory network. ARs with no stimuli/effectors described in the regulatory network were categorized as empty. We used these results to further curate and refine our model, and we report the results of this analysis prior to curation (V1) and after curation (V2). Here, we highlight some of the considerations we made during the process of manual curation, describing our analysis of several ARs as examples.

In some cases, our curation of the inconsistent ARs led to the removal of genes from the AR, which inconsistencies proved to be biologically meaningful. AR \#56 is one such example (Table 5). All members of AR \#56, except zur, respond to hydrogen peroxide as a stimuli; and upon further inspection, we note that AR \#56 corresponds almost perfectly with the hemAXCDBL operon (Hansson et al., 1991), which has been found to be regulated by PerR and is induced by hydrogen peroxide (Fuangthong et al., 2002). The inconsistent gene in AR \#56, Zur, is a PerR paralog 


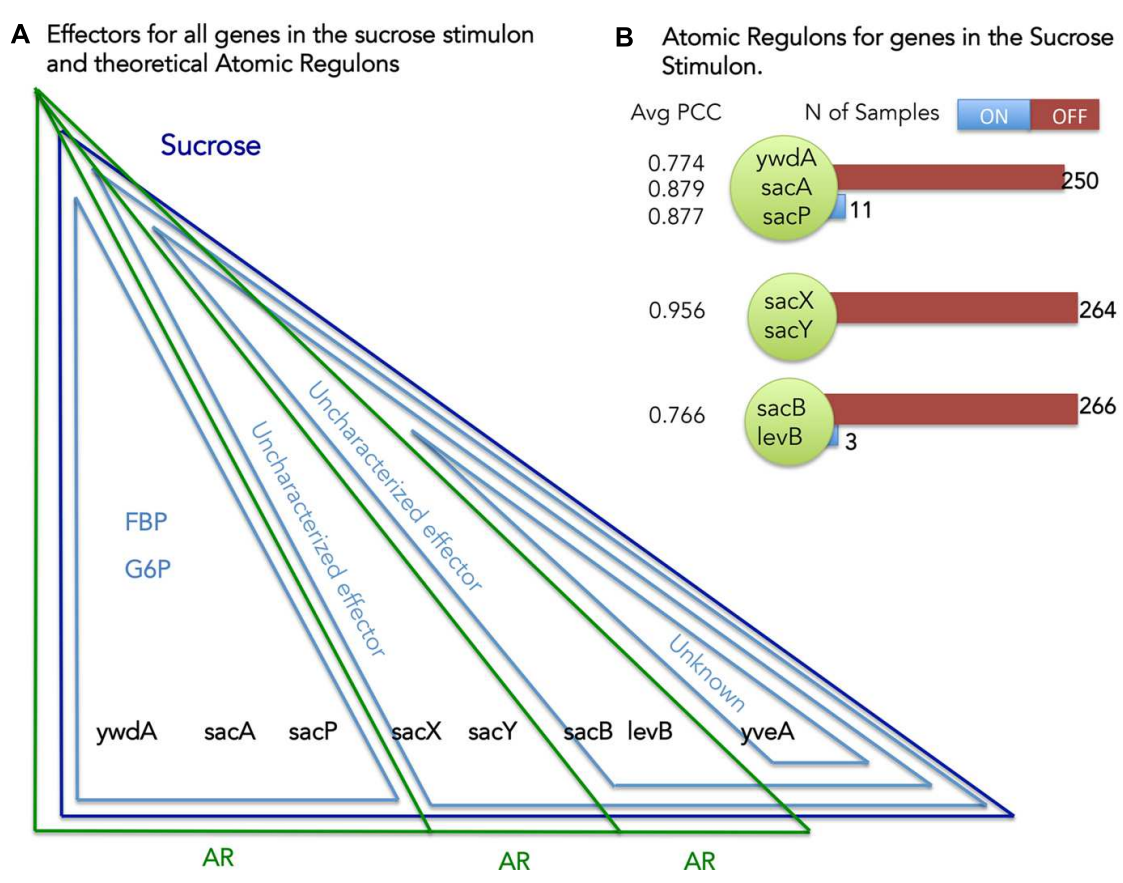

FIGURE 4 | Atomic Regulons (ARs) for the sucrose stimulon. (A) Effectors for all genes in the sucrose stimulon and theoretical ARs. Eight genes compose the sucrose stimulon (dark blue triangle). Fructose-biphospshate (FBP), Glucose-6-phosphate and uncharacterized effectors are also effectors (light blue triangles). The theoretical ARs are represented in green triangles. (B) ARs inferred for the sucrose stimulon. The ARs that were inferred are shown with the average Pearson correlation coefficient (PCC) and with a listing of the members of the AR. Number of samples ON and OFF for each AR is also shown.

(Fuangthong and Helmann, 2003) involved in regulation of the zinc homeostasis as the zinc uptake repressor (Gaballa et al., 2002). zur was removed from AR \#56, making this AR consistent according to our previously described crtieria. This finding also highlighted the fact that many of our ARs include regulatory genes in addition to regulatory gene target, which is undesirable for regulons being used within a regulatory network model. We thus used the information from our manually curated network to remove all known regulatory proteins from the ARs.
In other cases, our curation of the inconsistent $\mathrm{AR}$ aided us in identifying gene-stimuli interactions that were missing from our initial model. An example of this is AR \#612, which is comprised of 10 genes having functions associated with heme/iron transport (Table 6). From our regulatory network model, we have "Iron" associated with 8 out of the 10 AR members. A survey for yetG (now hmoA) revealed that the gene has been recently characterized to encode a heme-degrading monooxygenase (Gaballa and Helmann, 2011). hmoA has also

TABLE 2 | Sucrose stimulon represented in the Atomic Regulon (AR) web analysis resource.

\begin{tabular}{|c|c|c|}
\hline AR number* & Gene name/BSU & Stimuli \\
\hline 254 & $\operatorname{sacX}, \mathrm{BSU} 38410$ & Uncharacterized, Sucrose \\
\hline 254 & sacY, BSU38420 & Uncharacterized, Sucrose \\
\hline 376 & ywdA, BSU38030 & D-fructose-1,6-bisphosphate,Glucose-6-Phosphate, Sucrose \\
\hline 376 & sacA, BSU38040 & D-fructose-1,6-bisphosphate,Glucose-6-Phosphate, Sucrose \\
\hline 376 & sacP, BSU38050 & D-fructose-1,6-bisphosphate, Glucose-6-Phosphate, Sucrose \\
\hline 625 & sacB, BSU34450 & Uncharacterized, uncharacterized, Sucrose \\
\hline 625 & levB, BSU34460 & Uncharacterized, uncharacterized,Sucrose \\
\hline
\end{tabular}

*AR number arbitrarily assigned by the inference algorithm (the ARs described Figure 4 were assigned AR numbers 254, 376, and 625).

TABLE 3 | Experiments in which Atomic Regulon 625 was found to be "ON".

\begin{tabular}{|c|c|c|}
\hline Study & Sample & Study explanation \\
\hline study0003 & S6/t_2_hyb42359702 & Tested gene expression at regular intervals after sporulation was induced \\
\hline study0003 & S6_2_hyb29634602 & Tested gene expression at regular intervals after sporulation was induced \\
\hline study0003 & S8_5_hyb43271102 & Tested gene expression at regular intervals after sporulation was induced \\
\hline
\end{tabular}


TABLE 4 | Consistency of Atomic Regulons (ARs) with the regulatory network.

\begin{tabular}{lcc}
\hline Classification & V1 & V2 \\
\hline Consistent $(+)$ & 151 & 174 \\
Consistent with missing stimuli $( \pm)$ & 74 & 45 \\
Inconsistent $\left(^{*}\right)$ & 48 & 32 \\
Empty $(-)$ & 415 & 425 \\
Total & 688 & 676
\end{tabular}

Reflects the consistency of the original ARs (V1) and the curated ARs (V2). (+) All ARs members have the same stimuli/effectors in the regulatory network. ( \pm ) Some members of the AR have the same stimuli/effectors while other members have no described stimuli in the regulatory network. (*) ARs members have different stimuli associated in the regulatory network. (-) No stimuli described in the regulatory network.

TABLE 5 | Atomic Regulon 56.

\begin{tabular}{llc}
\hline Gene name / BSU & Stimuli & Average PCC \\
\hline zur, BSU25100 & & 0.664 \\
hemL, BSU28120 & Hydrogen peroxide & 0.885 \\
hemB, BSU28130 & Hydrogen peroxide & 0.900 \\
hemD, BSU28140 & Hydrogen peroxide & 0.905 \\
hemC, BSU28150 & Hydrogen peroxide & 0.906 \\
hemX, BSU28160 & Hydrogen peroxide & 0.893 \\
hemA, BSU28170 & Hydrogen peroxide & 0.804 \\
\hline
\end{tabular}

Stimuli and average Pearson correlation coefficient (PCC) among members of the $A R$ are shown for each gene (Gene names and B. subtilis specific BSU numbers provided) in the AR.

been shown to be regulated by Fur, the same regulator as the other members of AR \#612. There was no information on iron as stimuli for the yet $H$ gene product, and it had the lowest average PCC among all members of AR \#612. Despite those two facts, yet $H$ should remain part of the AR \#612 because the gene product is a member of the Glyoxalase/bleomycin resistance protein family and is known to be a metalloprotein. We verified this by using BLASTp against NCBI's CDD. This insight has been reflected in our B. subtilis SEED annotation by changing the functional role to "Glyoxalase/bleomycin resistance

TABLE 6 | Atomic Regulon 612.

\begin{tabular}{lcc}
\hline Gene name / BSU & Stimuli & Average PCC \\
\hline ycIN, BSU03800 & Iron & 0.804 \\
yclO, BSU03810 & Iron & 0.807 \\
ycIP, BSU03820 & Iron & 0.815 \\
yclQ, BSU03830 & Iron & 0.806 \\
yetG, BSU07150 & & 0.716 \\
yetH, BSU07160 & & 0.605 \\
yfmF, BSU07490 & Iron & 0.621 \\
yfmE, BSU07500 & Iron & 0.702 \\
yfmD, BSU07510 & Iron & 0.769 \\
yfmC, BSU07520 & Iron & 0.764 \\
yhfQ, BSU10330 & Iron & 0.779 \\
\hline Stimuli and average PCC among members of the AR are shown for each gene \\
(Gene names and B. subtilis specific BSU numbers provided) in the AR.
\end{tabular}

family metalloprotein". We suggest the expansion of regulatory information for $h m o A$ and yet $H$ to be consistent within AR \#612 with the addition of iron as stimuli and Fur as regulator (the latest release of Subtiwiki has already implemented one of the changes for $h m o A$ ).

Other inconsistencies involved genes in ARs where all members of the AR did not share the same set of stimuli. An example of this case is AR \#332 (Table 7). Three out of four members of the AR \#332 share the same effectors. These three members (treP, treA, and treR) were found to comprise the tre operon (Schock and Dahl, 1996). TreR is a transcriptional repressor, involved in the regulation of trehalose utilization and it is inhibited by trehalose-6-phosphate (Burklen et al., 1998). The additional stimuli, D-fructose-1,6-bisphosphate and Glucose6-Phosphate, relate to the activity of the carbon catabolite repression global regulator CcpA (Henkin, 1996). The fourth member of the $\mathrm{AR}, y f k O$, has been described in the literature as a nitroreductase (Prosser et al., 2010). Upon inspection of this region of the chromosome, we found $y f k O$ up-stream of the transcriptional regulator TreR, and not a member of the tre operon/TreR regulon. Due to this analysis we removed $y f k O$ from AR 332. As noted before, we also removed TreR as the protein imposing the regulatory activity. This curated AR was classified as "Trehalose Utilization".

Four hundred and fifteen ARs were found to have no associated stimuli in the regulatory network. Previously, we attempted to use details in the gene expression experiments to aid in the characterization of unknown effectors (Figure 4). We applied the same approach to ARs for which there are no effectors in the regulatory network, such as AR \#651 (Table 8). In addition to having no associated regulator or stimuli in the network, all genes in AR \#651 have unknown functions. The members of AR \#651 show a high average PCC and are only "ON" in a very small number of samples. In the experiment that activated AR \#651, cells were grown in $\mathrm{LB}$ medium at $37^{\circ} \mathrm{C}$ with vigorous shaking. During exponential growth (O.D.600 0.25), the cell culture was divided into two subcultures: one subculture acted as control [no mitomycin C, M0], while mitomycin was added to the second subculture at a concentration of $40 \mathrm{ng} / \mathrm{mL}$ [mitomycin, M40]. Samples were harvested at 0,45 and $90 \mathrm{~min}$ after mitomycin addition [t0, t45, and t90]. Addition of mitomycin $\mathrm{C}$ promoted prophage induction.

We used our web tools ${ }^{3}$ to identify all the genes that changed state between our control sample (grown in LB media only) and the sample grown with mitomycin $\mathrm{C}^{4}$. In the results, we see several $A R$ associated with prophages being $O N$ in the experiment wherein mitomycin $\mathrm{C}$ was added. Mitomycin $\mathrm{C}$ serves to stimulate the expression of these specific genes, leading to its addition as a stimulus for these ARs.

The "case studies" presented before were part a larger manual curation effort. All changes made to ARs during the curation process can be found in Supplementary Table S4. In Table 4 we can see the impact of the curation process (V2) across our four categories of $\mathrm{AR}$ consistency. We see a decrease in ARs

\footnotetext{
${ }^{3}$ http://tinyurl.com/ARStudies

${ }^{4}$ http://tinyurl.com/LBvsMitomycin
} 
TABLE 7 | Atomic Regulon 332.

\begin{tabular}{|c|c|c|c|}
\hline Gene name / BSU & Stimuli & Average PCC & Function \\
\hline treP,BSU07800 & $\begin{array}{l}\text { D-fructose-1,6-bisphosphate,Glucose-6- } \\
\text { Phosphate,phosphate,D-trehalose-6-phosphate }\end{array}$ & 0.807 & PTS system, trehalose-specific IIB component (EC 2.7.1.69) \\
\hline treA,BSU07810 & $\begin{array}{l}\text { D-fructose-1,6-bisphosphate,Glucose-6- } \\
\text { Phosphate,phosphate,D-trehalose-6-phosphate }\end{array}$ & 0.814 & Trehalose-6-phosphate hydrolase (EC 3.2.1.93) \\
\hline treR,BSU07820 & $\begin{array}{l}\text { D-fructose-1,6-bisphosphate,Glucose-6- } \\
\text { Phosphate,phosphate,D-trehalose-6-phosphate }\end{array}$ & 0.734 & Trehalose operon transcriptional repressor \\
\hline yfkO,BSU07830 & Disulfide_stress_conditions & 0.706 & Oxygen-insensitive NAD(P)H nitroreductase (EC 1.-.-.-) \\
\hline
\end{tabular}

Stimuli, average PCC among members of the AR and functional role is shown for each gene (Gene names and B. subtilis specific BSU numbers provided) in the AR.

TABLE 8| Atomic Regulon 651.

\begin{tabular}{lccccc}
\hline Gene name / BSU & Stimuli & Average PCC & Function & OFF & ON \\
\hline yosW,BSU19980 & - & 0.906 & unknown & 261 & 4 \\
yosV,BSU19990 & - & 0.948 & unknown & 261 & 4 \\
yojW,BSU19999 & - & 0.941 & unknown & 261 & 4 \\
\hline
\end{tabular}

Stimuli, average PCC among members of the AR and functional role are shown for each gene (Gene names and B. subtilis specific BSU numbers provided) in the AR.

categorized as inconsistent and consistent with missing stimuli. This led to a subsequent increase in the number of consistent ARs. The improved set (Supplementary Tables S5 and S6) contains new versions of ARs and the Regulatory network that reflect all the changes made during the manual curation process.

\section{CONCLUSION}

We introduce a new, expanded regulatory network for B. subtilis, compiling information from multiple notable sources of gene regulatory data as well as our own inferences. We show that our reconstruction is more comprehensive than other previous versions found in the literature. This new network is a valuable resource of known regulatory interactions occurring within B. subtilis 168, and it may be readily integrated with existing genome-scale metabolic models of B. subtilis (Henry et al., 2009; Tanaka et al., 2013) to improve model predictions by accounting for regulatory interactions (Covert and Palsson, 2002; Herrgard et al., 2004). We demonstrate through our analysis of the model the prevalence of regulators responding to small molecule effectors. We also show the prevalence of sigma-factor regulation in $B$. subtilis, in terms of number of impacted genes. Finally, we explore how the most complex regulation is used to trigger major system responses to a wide range of environmental conditions.

Additionally, we introduce the use of ARs to reconcile our proposed network with available gene expression data. We show how this methodology is able to fill gaps and correct inconsistencies in the regulatory network. The reconciliation process allowed us to expand our knowledge of $B$. subtilis regulation by adding new stimuli to genes and regulons, by adjusting stimuli on existing genes, and by adjust regulons based on inconsistent expression patterns. We were also able to provide clues for putative gene function assignments for genes with unknown functions.

All AR data were integrated into the PubSEED ${ }^{5}$, and they can be used as part of the annotation curation tools available in that framework. A web resource was also created showing the relationship between the ARs and the expression data used for their computation in B. subtilis. This resource is available for the public and can be used to conduct analysis of the ARs and expression data sets beyond the objectives of the work described in this manuscript.

During the reconciliation process, we were able to match many ARs to the same regulatory mechanisms we found in our manually curated network. This fact highlights the convenience of using ARs to study the regulatory network of an organism without a huge effort put into initial manual curation.

\section{AUTHOR CONTRIBUTIONS}

JF, AG, and VF contributed to the regulatory network reconstruction manual curation efforts from notable Bacilus subtilis regulation resources. The inference of Atomic Regulons was performed by JF, RO, and RT. JF, VV, and RO performed the reconciliation of the regulatory network with the gene expression data. JF, MR, IR, and $\mathrm{CH}$ contributed to the analysis of the new reconciled regulatory network. $\mathrm{RO}$ and $\mathrm{NC}$ contributed to the web resources introduced in the manuscript. All authors contributed to data analysis and manuscript preparation. $\mathrm{CH}$ conceived of the project and oversaw work.

\section{FUNDING}

JF acknowledges funding from [FRH/BD/70824/2010] of the FCT (Portuguese Foundation for Science and Technology) Ph.D. program. CH was supported by the National Science Foundation under grant number EFRI-MIKS-1137089 and the US Department of Energy under contract DE-ACO206CH11357. RT was supported by the Genomic Science Program (GSP), Office of Biological and Environmental Research (OBER), U.S. Department of Energy (DOE), and his work is a contribution of the Pacific Northwest National Laboratory (PNNL) Foundational Scientific Focus Area.

\footnotetext{
${ }^{5} \mathrm{http}: / /$ pubseed.theseed.org/
} 


\section{ACKNOWLEDGMENT}

AG and VF acknowledge funding from the European Union Basynthec project (BaSynthec FP7-244093).

\section{SUPPLEMENTARY MATERIAL}

The Supplementary Material for this article can be found online at: http://journal.frontiersin.org/article/10.3389/fmicb. 2016.00275

\section{REFERENCES}

Anagnostopoulos, C., and Spizizen, J. (1961). Requirements for transformation in Bacillus subtilis. J. Bacteriol. 81, 741-746.

Buescher, J. M., Liebermeister, W., Jules, M., Uhr, M., Muntel, J., Botella, E., et al. (2012). Global network reorganization during dynamic adaptations of Bacillus subtilis metabolism. Science 335, 1099-1103. doi: 10.1126/science.1206871

Burklen, L., Schock, F., and Dahl, M. K. (1998). Molecular analysis of the interaction between the Bacillus subtilis trehalose repressor TreR and the tre operator. Mol. Gen. Genet. 260, 48-55. doi: 10.1007/s004380050869

Chen, K., and Rajewsky, N. (2007). The evolution of gene regulation by transcription factors and microRNAs. Nat. Rev. Genet. 8, 93-103. doi: $10.1038 /$ nrg1990

Cho, B. K., Zengler, K., Qiu, Y., Park, Y. S., Knight, E. M., Barrett, C. L., et al. (2009). The transcription unit architecture of the Escherichia coli genome. Nat. Biotechnol. 27, 1043-1049. doi: 10.1038/nbt.1582

Commichau, F. M., Pietack, N., and Stulke, J. (2013). Essential genes in Bacillus subtilis: a re-evaluation after ten years. Mol. Biosyst. 9, 1068-1075. doi: $10.1039 / \mathrm{c} 3 \mathrm{mb} 25595 \mathrm{f}$

Covert, M. W., and Palsson, B. O. (2002). Transcriptional regulation in constraintsbased metabolic models of Escherichia coli. J. Biol. Chem. 277, 28058-28064. doi: 10.1074/jbc.M201691200

de Hoon, M. J., Imoto, S., Kobayashi, K., Ogasawara, N., and Miyano, S. (2003). Inferring gene regulatory networks from time-ordered gene expression data of Bacillus subtilis using differential equations. Pac. Symp. Biocomput. 8, 17-28.

Debarbouille, M., Arnaud, M., Fouet, A., Klier, A., and Rapoport, G. (1990). The sacT gene regulating the sacPA operon in Bacillus subtilis shares strong homology with transcriptional antiterminators. J. Bacteriol. 172, 3966-3973.

Edgar, R., Domrachev, M., and Lash, A. E. (2002). Gene expression omnibus: NCBI gene expression and hybridization array data repository. Nucleic Acids Res. 30, 207-210. doi: 10.1093/nar/30.1.207

Eijlander, R. T., de Jong, A., Krawczyk, A. O., Holsappel, S., and Kuipers, O. P. (2014). SporeWeb: an interactive journey through the complete sporulation cycle of Bacillus subtilis. Nucleic Acids Res. 42, D685-D691. doi: 10.1093/nar/gkt1007

Ermolaeva, M. D., White, O., and Salzberg, S. L. (2001). Prediction of operons in microbial genomes. Nucleic Acids Res. 29, 1216-1221. doi: 10.1093/nar/29.5.1216

Fadda, A., Fierro, A. C., Lemmens, K., Monsieurs, P., Engelen, K., and Marchal, K. (2009). Inferring the transcriptional network of Bacillus subtilis. Mol. Biosyst. 5, 1840-1852. doi: 10.1039/b907310h

Florez, L. A., Roppel, S. F., Schmeisky, A. G., Lammers, C. R., and Stulke, J. (2009). A community-curated consensual annotation that is continuously updated: the Bacillus subtilis centred wiki SubtiWiki. Database (Oxford) 2009:ba012. doi: 10.1093/database/bap012

Fuangthong, M., and Helmann, J. D. (2003). Recognition of DNA by three ferric uptake regulator (Fur) homologs in Bacillus subtilis. J. Bacteriol. 185, 63486357. doi: 10.1128/JB.185.21.6348-6357.2003

Fuangthong, M., Herbig, A. F., Bsat, N., and Helmann, J. D. (2002). Regulation of the Bacillus subtilis fur and perR genes by PerR: not all members of the PerR regulon are peroxide inducible. J. Bacteriol. 184, 3276-3286. doi: 10.1128/JB.184.12.3276-3286.2002
TABLE S1 | Manually curated regulatory network for Bacillus subtilis.

TABLE S2 | Regulators described in the regulatory network.

TABLE S3 | Atomic Regulons for B. subtilis.

TABLE S4 | Curation of Atomic Regulons.

TABLE S5 | Atomic Regulons or B. subtilis (version 2).

TABLE S6 | Manually curated regulatory network for B. subtilis (version 2).

Fujita, Y. (2009). Carbon catabolite control of the metabolic network in Bacillus subtilis. Biosci. Biotechnol. Biochem. 73, 245-259. doi: 10.1271/ bbb. 80479

Gaballa, A., and Helmann, J. D. (2011). Bacillus subtilis Fur represses one of two paralogous haem-degrading monooxygenases. Microbiology 157, 3221-3231. doi: 10.1099/mic.0.053579-0

Gaballa, A., Wang, T., Ye, R. W., and Helmann, J. D. (2002). Functional analysis of the Bacillus subtilis Zur regulon. J. Bacteriol. 184, 6508-6514. doi: 10.1128/JB.184.23.6508-6514.2002

Goelzer, A., Bekkal Brikci, F., Martin-Verstraete, I., Noirot, P., Bessieres, P., Aymerich, S., et al. (2008). Reconstruction and analysis of the genetic and metabolic regulatory networks of the central metabolism of Bacillus subtilis. BMC Syst. Biol. 2:20. doi: 10.1186/1752-0509-2-20

Guell, M., van Noort, V., Yus, E., Chen, W. H., Leigh-Bell, J., Michalodimitrakis, K., et al. (2009). Transcriptome complexity in a genome-reduced bacterium. Science 326, 1268-1271. doi: 10.1126/science.1176951

Hansson, M., Rutberg, L., Schroder, I., and Hederstedt, L. (1991). The Bacillus subtilis hemAXCDBL gene cluster, which encodes enzymes of the biosynthetic pathway from glutamate to uroporphyrinogen III. J. Bacteriol. 173, 2590-2599.

Henkin, T. M. (1996). The role of CcpA transcriptional regulator in carbon metabolism in Bacillus subtilis. FEMS Microbiol. Lett. 135, 9-15. doi: 10.1111/j.1574-6968.1996.tb07959.x

Henry, C. S., Zinner, J. F., Cohoon, M. P., and Stevens, R. L. (2009). iBsu1103: a new genome-scale metabolic model of Bacillus subtilis based on SEED annotations. Genome Biol. 10:R69. doi: 10.1186/gb-2009-10-6-r69

Herrgard, M. J., Covert, M. W., and Palsson, B. O. (2004). Reconstruction of microbial transcriptional regulatory networks. Curr. Opin. Biotechnol. 15, 7077. doi: 10.1016/j.copbio.2003.11.002

Illing, N. (2002). Bacillus subtilis and its closest relatives: from genes to cells. Nature 415, 263-264. doi: 10.1038/415263b

Kobayashi, K., Ehrlich, S. D., Albertini, A., Amati, G., Andersen, K. K., Arnaud, M., et al. (2003). Essential Bacillus subtilis genes. Proc. Natl. Acad. Sci. U.S.A. 100, 4678-4683. doi: 10.1073/pnas.0730515100

Leyn, S. A., Kazanov, M. D., Sernova, N. V., Ermakova, E. O., Novichkov, P. S., and Rodionov, D. A. (2013). Genomic reconstruction of the transcriptional regulatory network in Bacillus subtilis. J. Bacteriol. 195, 2463-2473. doi: 10.1128/JB.00140-13

Lopez, D., and Kolter, R. (2010). Extracellular signals that define distinct and coexisting cell fates in Bacillus subtilis. FEMS Microbiol. Rev. 34, 134-149. doi: 10.1111/j.1574-6976.2009.00199.x

Mader, U., Schmeisky, A. G., Florez, L. A., and Stulke, J. (2012). SubtiWiki-a comprehensive community resource for the model organism Bacillus subtilis. Nucleic Acids Res. 40, D1278-D1287. doi: 10.1093/nar/gkr923

Marchler-Bauer, A., Derbyshire, M. K., Gonzales, N. R., Lu, S., Chitsaz, F., Geer, L. Y., et al. (2015). CDD: NCBI's conserved domain database. Nucleic Acids Res. 43, D222-D226. doi: 10.1093/nar/gku1221

Medini, D., Serruto, D., Parkhill, J., Relman, D. A., Donati, C., Moxon, R., et al. (2008). Microbiology in the post-genomic era. Nat. Rev. Microbiol. 6, 419-430. doi: 10.1038/nrmicro1901

Michna, R. H., Commichau, F. M., Todter, D., Zschiedrich, C. P., and Stulke, J. (2014). SubtiWiki-a database for the model organism Bacillus subtilis that 
links pathway, interaction and expression information. Nucleic Acids Res. 42, D692-D698. doi: 10.1093/nar/gkt1002

Mironov, A. S., Gusarov, I., Rafikov, R., Lopez, L. E., Shatalin, K., Kreneva, R. A., et al. (2002). Sensing small molecules by nascent RNA: a mechanism to control transcription in bacteria. Cell 111, 747-756. doi: 10.1016/S0092-8674(02) 01134-0

Nicolas, P., Mader, U., Dervyn, E., Rochat, T., Leduc, A., Pigeonneau, N., et al. (2012). Condition-dependent transcriptome reveals high-level regulatory architecture in Bacillus subtilis. Science 335, 1103-1106. doi: 10.1126/science. 1206848

Novichkov, P. S., Laikova, O. N., Novichkova, E. S., Gelfand, M. S., Arkin, A. P., Dubchak, I., et al. (2010a). RegPrecise: a database of curated genomic inferences of transcriptional regulatory interactions in prokaryotes. Nucleic Acids Res. 38, D111-D118. doi: 10.1093/nar/gkp894

Novichkov, P. S., Rodionov, D. A., Stavrovskaya, E. D., Novichkova, E. S., Kazakov, A. E., Gelfand, M. S., et al. (2010b). RegPredict: an integrated system for regulon inference in prokaryotes by comparative genomics approach. Nucleic Acids Res. 38, W299-W307. doi: 10.1093/nar/gkq531

Nudler, E., and Mironov, A. S. (2004). The riboswitch control of bacterial metabolism. Trends Biochem. Sci. 29, 11-17. doi: 10.1016/j.tibs.2003.11.004

Overbeek, R., Begley, T., Butler, R. M., Choudhuri, J. V., Chuang, H. Y., Cohoon, M., et al. (2005). The subsystems approach to genome annotation and its use in the project to annotate 1000 genomes. Nucleic Acids Res. 33, 5691-5702. doi: 10.1093/nar/gki866

Pereira, Y., Petit-Glatron, M. F., and Chambert, R. (2001). yveB, Encoding endolevanase LevB, is part of the sacB-yveB-yveA levansucrase tricistronic operon in Bacillus subtilis. Microbiology 147, 3413-3419. doi: 10.1099/00221287-147-12-3413

Price, M. N., Huang, K. H., Alm, E. J., and Arkin, A. P. (2005). A novel method for accurate operon predictions in all sequenced prokaryotes. Nucleic Acids Res. 33, 880-892. doi: 10.1093/nar/gki232

Prosser, G. A., Patterson, A. V., and Ackerley, D. F. (2010). uvrB gene deletion enhances SOS chromotest sensitivity for nitroreductases that preferentially generate the 4-hydroxylamine metabolite of the anti-cancer prodrug CB1954. J. Biotechnol. 150, 190-194. doi: 10.1016/j.jbiotec.2010.08.007
Schock, F., and Dahl, M. K. (1996). Expression of the tre operon of Bacillus subtilis 168 is regulated by the repressor TreR. J. Bacteriol. 178, 4576-4581.

Sierro, N., Makita, Y., de Hoon, M., and Nakai, K. (2008). DBTBS: a database of transcriptional regulation in Bacillus subtilis containing upstream intergenic conservation information. Nucleic Acids Res. 36, D93-D96. doi: 10.1093/nar/gkm910

Steggles, L. J., Banks, R., Shaw, O., and Wipat, A. (2007). Qualitatively modelling and analysing genetic regulatory networks: a Petri net approach. Bioinformatics 23, 336-343. doi: 10.1093/bioinformatics/btl596

Sterlini, J. M., and Mandelstam, J. (1969). Commitment to sporulation in Bacillus subtilis and its relationship to development of actinomycin resistance. Biochem. J. 113, 29-37. doi: 10.1042/bj1130029

Tanaka, K., Henry, C. S., Zinner, J. F., Jolivet, E., Cohoon, M. P., Xia, F., et al. (2013). Building the repertoire of dispensable chromosome regions in Bacillus subtilis entails major refinement of cognate large-scale metabolic model. Nucleic Acids Res. 41, 687-699. doi: 10.1093/nar/gks963

Tortosa, P., and Le Coq, D. (1995). A ribonucleic antiterminator sequence (RAT) and a distant palindrome are both involved in sucrose induction of the Bacillus subtilis sacXY regulatory operon. Microbiology 141(Pt 11), 2921-2927. doi: 10.1099/13500872-141-11-2921

Wagner, E. G., and Simons, R. W. (1994). Antisense RNA control in bacteria, phages, and plasmids. Annu. Rev. Microbiol. 48, 713-742. doi: 10.1146/annurev.mi.48.100194.003433

Conflict of Interest Statement: The authors declare that the research was conducted in the absence of any commercial or financial relationships that could be construed as a potential conflict of interest.

Copyright (C) 2016 Faria, Overbeek, Taylor, Conrad, Vonstein, Goelzer, Fromion, Rocha, Rocha and Henry. This is an open-access article distributed under the terms of the Creative Commons Attribution License (CC BY). The use, distribution or reproduction in other forums is permitted, provided the original author(s) or licensor are credited and that the original publication in this journal is cited, in accordance with accepted academic practice. No use, distribution or reproduction is permitted which does not comply with these terms. 